\title{
RÉFUGIÉS ET SITUATIONS CONFLICTUELLES
}

La protection des réfugiés et des personnes déplacées est assurée par de nombreux instruments juridiques internationaux, universels et régionaux. L'appareil normatif existe mais, depuis plusieurs années, les organisations humanitaires chargées de le mettre en ouvre doivent faire face sans cesse à des situations nouvelles causées par l'ampleur et la fréquence d'exodes massifs de populations, notamment dans le Tiers Monde et à des nouveaux phénomènes de violence qui affectent ces populations dans leur statut comme dans leur protection. Et l'on a pu constater très souvent que les solutions apportées par les organisations humanitaires chargées de la protection des réfugiés se mesuraient en terme d'assistance plutôt qu'en terme de protection sans que l'on puisse toujours déterminer clairement la ligne de démarcation entre ces deux domaines.

Dès lors, dans quelle mesure les normes juridiques assurent-elles la protection aux réfugiés dits traditionnels et aux personnes qui, sans être des réfugiés, sont forcées de quitter leur pays pour cause de conflit armé ou de troubles intérieurs graves? Comment les organismes humanitaires de protection et d'assistance se sont-ils adaptés aux réalités, dans le domaine qui nous préoccupe?

En outre, la complexité croissante des problèmes des réfugiés et des personnes déplacées, notamment dans les situations conflictuelles, requiert une coopération toujours plus étroite entre les organismes humanitaires chargés de leur protection et de leur assistance. Quel diagnostic peut-on porter en 1988 sur les compétences respectives de ces organisations, sur la complémentarité de leur action et sur le degré de leur coopération?

La Revue internationale de la Croix-Rouge, en retenant ce thème de "Réfugiés et situations conflictuelles" pour le présent numéro, s'est adressée au Haut Commissaire des Nations Unies pour les réfugiés, au Président de l'Institut international de droit humanitaire, à la Division juridique du CICR et à un professeur de droit de l'Université Chula- 
longkorn de Bangkok et les a invités à partager avec ses lecteurs leurs analyses et leurs réflexions sur ces questions qui ne cessent de préoccuper la communauté internationale.

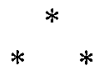

Dans son article «Protéger par l'action», M. Jean-Pierre Hocké, Haut Commissaire des Nations Unies pour les réfugiés, axant sa réflexion sur l'évolution de l'action de cette institution créée en 1951, constate que la problématique des réfugiés est telle que la protection de ces derniers ne peut plus être assurée si elle n'est pas assortie d'une assistance. En vérité, l'intégration des fonctions de protection et d'assistance est devenue graduellement la condition nécessaire à la réalisation du mandat du HCR.

Cette conjonction de la protection et de l'assistance constitue déjà la spécificité de la Croix-Rouge dans les conflits armés. Ces deux concepts sont et doivent rester indissociables, condition essentielle pour que soient assurés la défense des victimes des conflits armés, le respect de la vie et de la dignité humaine ${ }^{1}$.

Cette similitude de l'action de protection et d'assistance du HCR et du CICR repose sur des fondements juridiques qu'il n'est pas inutile de rappeler et surtout d'analyser à la lumière des changements sociopolitiques de ces dernières années.

L'action du CICR en faveur des réfugiés est analysée par Françoise Krill, membre de la Division juridique du CICR. Son étude juridique détaillée couvre tous les cas de figure des situations du réfugié considéré au regard du droit international humanitaire comme une personne civile protégée à ce titre par le DIH et selon la systématique prévue par ce droit pour la protection des personnes civiles en temps de conflits armés. Elle montre particulièrement les progrés réalisés par le Protocole I de 1977 dans la protection du réfugié, soit personne civile au pouvoir d'une Partie au conflit, soit personne civile victime des effets des hostilités. Tel est notamment le cas dans le contexte des attaques militaires de camps de réfugiés.

L'auteur développe également les bases juridiques de l'action du $C I C R$ en faveur des réfugiés pour mieux en dégager la spécificité: le $C I C R$ entreprend une action en faveur des réfugiés quand l'existence de ces derniers est liée à un conflit; d'où la compétence principale dont jouit le CICR pour la protection des personnes déplacées à l'intérieur d'un pays qui vit une telle situation.

1 Voir à ce sujet Jean-Luc Blondel, «L'assistance aux personnes protégées», Revue internationale de la Croix-Rouge (RICR), $\mathrm{n}^{\circ} 767$, septembre-octobre 1987, pp. 471-489. 
Cet article annonce judicieusement la contribution du professeur Vitit Muntarbhorn de l'Université Chulalongkorn de Bangkok, lequel procède à une analyse des mandats du Mouvement international de la CroixRouge et du Croissant-Rouge, notamment du CICR et du HCR dans le domaine de la protection et de l'assistance des réfugiés en cas de conflits armés ou de troubles intérieurs: il en dégage les similitudes, les recoupements et les différences. Mais cette analyse s'accompagne d'une réflexion sur l'évolution des mandats respectifs du CICR et du HCR, compte tenu des nouvelles formes de conflits et de troubles. A son tour l'auteur met l'accent sur la complémentarité des tâches des deux institutions: si le HCR a vu son mandat s'étendre aux personnes déplacées hors de leur pays d'origine, il ne couvre pas encore, au sens strict du droit, les personnes déplacées à l'intérieur de leur pays d'origine, ce qui, par contre, entre dans les compétences du CICR en cas de conflits armés et de troubles interieurs.

Reste le cas des situations marginales, telles celles des réfugiés dans les zones frontalières en butte à des attaques armées. Là, le droit est flou quant aux compétences respectives du HCR et du CICR. Mais un progrès sensible a été réalisé lors de la XXIVe Conférence internationale de la Croix-Rouge en 1981: une résolution a été adoptée qui clarifie la situation en soulignant le caractère complémentaire des activités des deux institutions et en les engageant à se consulter et à coordonner leur action d'assistance 2 .

Malgré ces progrés réels, l'auteur estime souhaitable, afin de parer aux divergences qui peuvent apparaître sur le sens des termes de "réfugié» ou de "personne déplacée» et de faire face à toute situation nouvelle dont les victimes n'entrent pas dans une catégorie bien définie mais requièrent protection et assistance, que soit élaboré un ensemble de principes de base en matière de protection et d'assistance humanitaires, basés sur la législation actuelle et sur la pratique. Ces principes serviraient de lignes directrices pour les opérations humanitaires.

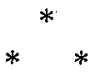

Cette complémentarité du droit humanitaire et du droit des réfugiés a fait également l'objet d'études au sein de l'Institut international de droit humanitaire. Son président, le professeur Jovića Patrnogic, démonte les rouages du droit humanitaire, ce droit bien établi, et du droit des réfugiés, ce droit encore peu développé dont il souhaite une codification plus rigoureuse au vu des problèmes du temps.

${ }^{2}$ XXIVe Conférence internationale de la Croix-Rouge, Manille, 1981, résolution $\mathrm{XXI}$, «Action de la Croix-Rouge internationale en faveur des réfugiés». 
S'il reconnait volontiers que l'évolution de ce qu'il appelle les droits fondamentaux humains: droit des droits de l'homme, droit international humanitaire, droit international des réfugiés, est encourageante, force est de constater que leur respect se heurte à plusieurs obstacles majeurs au premier rang desquels il mentionne l'interprétation excessive de la notion de souveraineté de l'Etat, l'érosion du principe du non-refoulement sans oublier les cas de violations graves du droit affectant réfugiés et personnes déplacées.

Dès lors, la question des relations délicates entre le politique et l'humanitaire pèse de tout son poids. Comme le dit J. P. Hocké, "s'il ne peut $y$ avoir de solution humanitaire sans solution politique, il peut, et doit, $y$ avoir action humanitaire en attendant la solution politique. Dans cette perspective, l'action humanitaire justement parce qu'elle n'est pas politique, peut contribuer à créer les conditions d'une solution politique». L'action humanitaire, celle du CICR, celle du HCR, pour ne citer que ces deux institutions, devient un combat permanent afin qu'elle puisse influencer le politique et créer les conditions d'une solution politique. On pourrait citer ici à juste titre le président du CICR lorsqu'à l'ouverture de la Seconde Conférence mondiale de la Croix-Rouge et du CroissantRouge sur la paix (Aaland/Stockholm, 1984), il proclamait: «...L'action humanitaire ne saurait en elle même prétendre apporter de solutions définitives aux problèmes fondamentaux qui sont à l'origine des conflits, puisque ceux-ci sont politiques et que, par là même, ils lui échappent. Toutefois, cette même action humanitaire, bien comprise et «intelligemment utilisée " par les gouvernements, peut donner à ces derniers un temps de répit, qu'ils devraient mettre à profit pour chercher, dans un esprit de paix et de conciliation, les solutions politiques qui, à leur tour, feront disparaître les problèmes humanitaires». ${ }^{3}$

Comme le souligne le professeur Patrnogic, une stratégie humanitaire s'impose, plus que jamais; elle est déjà dessinée par le caractère complémentaire des mandats et des activités du CICR et du HCR, elle est illustrée par la promotion des instruments internationaux humanitaires, par la diffusion méthodique des principes de base du droit humanitaire et du droit des réfugiés, le développement continu des normes du droit adaptées aux besoins nouveaux. La garantie de la protection des réfugiés et des victimes des conflits armés et autres situations conflictuelles est à ce prix.

La Revue

3 «Message inaugural à la Seconde Conférence mondiale de la Croix-Rouge et du Croissant-Rouge sur la paix» par Alexandre Hay, président du CICR, RICR, $\mathrm{n}^{\circ} 750$, novembre-décembre 1984 , p. 344. 